\title{
Ion Association in Aqueous Solution
}

\author{
Marielle Soniat, Grayson Pool, Lisette Franklin, and Steven W. Rick* \\ Department of Chemistry, University of New Orleans, New Orleans, LA, 70148, ${ }^{*}$ E-mail: \\ srick@uno.edu
}

\begin{abstract}
The properties of aqueous ionic solutions are determined by strong electrostatic interactions, which are influenced by polarizability and charge transfer interactions. Potential models which include polarizability and charge transfer have been developed for water and single ion properties. Here, the ion-ion interactions are optimized so that the osmotic pressure as a function of concentration is reproduced. Using the optimized potentials, the amount of ion pairing and larger cluster formation is calculated. For $\mathrm{NaCl}, \mathrm{NaI}$, and $\mathrm{KCl}$, there is a small amount of pairing, with larger clusters present as well. For KI, there is much more pairing and much larger clusters are observed. The amount of pairing is consistent with the law of matching affinities, with the pairs that show the least pairing also being the most mis-matched in terms of size or solvation free energy. The charge transfered from the anions to water is more than from is transferred from the water to cations, so the water molecules acquire a negative charge, which increases with ion concentration.
\end{abstract}

Keywords: Ionic solutions, charge transfer, molecular simulations, ion pairing, osmotic coefficient 


\title{
Ion Association in Aqueous Solution
}

\author{
Marielle Soniat, Grayson Pool, Lisette Franklin, and Steven W. Rick* \\ Department of Chemistry, University of New Orleans, New Orleans, LA, 70148, * E-mail: \\ srick@uno.edu
}

\section{Introduction}

The aqueous hydration of ions involves a balance of ion-ion, ion-water interactions, and water-water interactions, in order of decreasing strength. For example, the interaction energy between a sodium and a chloride ion is $-132 \mathrm{kcal} / \mathrm{mol},[1]$ the water-chloride interaction energy is $-13.6 \mathrm{kcal} / \mathrm{mol}[2]$ and the water-water interaction energy is $-5.0 \mathrm{kcal} / \mathrm{mol}$.[3] This balance of large energies is indicated by the large lattice energy of salts $(188 \mathrm{kcal} / \mathrm{mol}$ for $\mathrm{NaCl})$ and the large solvation enthalpy $(-187 \mathrm{kcal} / \mathrm{mol}$ for $\mathrm{NaCl})$ adding up to give a relatively small heat of solution of about $1 \mathrm{kcal} / \mathrm{mol}$.[4] The interplay of these interactions can determine if a salt is insoluable, completely dissociated in solution, or some intermediate point involving ion pairing. Experimental evidence for ion pairing for univalent ions below supersaturation comes from a variety of sources. Ion pairing is indicated in a variety of other approaches, including conductometry,[5] potentiometry,[6] ultrasonic relaxation,[7] near edge X-ray absorption spectrocopy,[8] dielectric relaxation spectroscopy, [9] and near infrared spectroscopy,[10] (see Reference [11]), and molecular dynamics simulations. [12, 13, 14, 15, 16, 17, 18, 19, 20, 21, 22, 23] The most direct structural indication of pairing would come from neutron diffraction or X-ray scattering but the ion-ion signal in both approaches is small relative to the signal from water-water and water-ion correlations, and so these approaches rely on molecular dynamics simulations to determine the amount of ion pairing. [24, 25] Clusters larger than pairs are found for solutions under ambient conditions at unsaturated concentrations from Raman[26] and vibrational energy transfer[27] experiments as well as molecular dynamics simulations.[28, 29, 30, 31, 32] The simulations revealed that alkali halide salts can form clusters of up to about five ions and while neutral aggregates are most probable, charged clusters are also found, with a preference for excess cations. [30, 32] The smaller cations tend to cluster more than larger cations. [30] Experiments on alkali-thiocyanate salts reveal a different picture. The charged clusters in those solutions tend to have an excess of anions and the bigger cations tend to cluster more, suggesting that cluster formation might be driven by the matching of cation and anion types.[27]

Osmotic pressure can indicate the degree of ion pairing.[11] In a concentrated solution, water-water interaction and interactions between unlike ions tend to pro- 
mote phase separation into a very concentrated solution and pure water, whereas ion-water and like-ion interactions favor a single dilute solution. If ions in a concentrated solution are restrained by a semi-permeable membrane, the pressure exerted by the solution may be less than expected for an ideal solution of fully dissolved salt. Reproduction of the osmotic pressure for salt solutions has been used to parameterize potential models for ion interactions.[17, 33, 34, 21, 23] Other related methods use the activity coefficient for ion-pair potential refinement.[35, 36, 37] These efforts show that changing only the unlike ion interaction, thereby changing the amount of ion pairing, and leaving all other interactions unchanged has a strong effect on the osmotic pressure. [34, 21, 23] This method is effective because small changes in the energy can shift the balance between the contact ion pair and the fully dissociated ions. Ion pairing is believed to be largely driven by the strength of the water-ion interaction, as given by the law of matching water affinities.[38] The osmotic coefficient shows a correlation with the difference in the absolute free energy of solution between the anion and the cation (Figure 1, data from References [39, 40,41]). This shows a similar volcano relationship (or in this case an inverted volcano) to other thermodynamic properties.[38] The salts that have the smallest osmotic coefficient are those with the closest free energies of solution. This includes large anion-large cation pairs like CsI (which has the lowest osmotic coefficient among the salts in Figure 1) and small anion-small cation pairs like NaF. Both small anion-large cation (on the left of Figure 1) and large anion-small cation (on the right) have larger osmotic pressures.

In this paper, we use recently developed models which include charge transfer and polarizability $[42,43,44]$ to examine the formation of ion pairs and larger clusters. Models for $\mathrm{Cl}^{-}, \mathrm{I}^{-}, \mathrm{Na}^{+}$, and $\mathrm{K}^{+}$have been developed which reproduce the single ion free energies of solution as well as other structural, energetic, and dynamical properties.[43, 44] We will adjust the interactions between unlike ion pairs to reproduce the osmotic coefficient as a function of concentration as has been done in previous studies. [34, 21, 23] The optimized potentials will be used for the clustering analysis of the four salt solutions, $\mathrm{NaCl}, \mathrm{NaI}, \mathrm{KCl}$, and $\mathrm{KI}$.

\section{Methods}

The osmotic pressure calculations. The osmotic pressure was calculated using the method of Luo and Roux[21] in which an artificial semi-permeable membrane is set up, which is permeable to water but not to ions. This method has the virtues of being exact, and so applicable to solutions of any concentration, and easy to implement. This is done by simply setting up a half-harmonic restraint which only acts on the $\mathrm{z}$ component of the ions according to

$$
E_{\text {restraint }}= \begin{cases}0 & z<z_{r} \\ \frac{1}{2} k_{r}\left(z-z_{r}\right)^{2} & z \geq z_{r}\end{cases}
$$


where $k_{r}$ is the force constant (set equal to $10 \mathrm{kcal} / \mathrm{mol} / \AA^{2}$ ) and $z_{r}$ is the position of the restraining membrane (set equal to $22 \AA$ ). There are two such restraining potentials, at $\pm z_{r}$, which creates a layer $2 z_{r}$ thick containing the salt solution. Outside this layer is pure water. The osmotic pressure is simply the force, $-k_{r}\left(z-z_{r}\right)$, that the restraint exerts on the ion divided by the area of the periodic cell in the $\mathrm{x}$ and $\mathrm{y}$ directions. The osmotic coefficient, $\phi$, is the osmotic pressure divided by the ideal solution osmotic pressure, $2 \mathrm{c}(\mathrm{m}) \mathrm{RT}$, where $\mathrm{c}(\mathrm{m})$ is the molality of the salt solution. Notice that the factor of 2 indicates that the ideal solution limit assumes complete ion dissociation. Ion pairing would then tend to decrease the osmotic pressure by having fewer independent particles. These simulations used an orthorhombic box with side $\mathrm{x}$ and $\mathrm{y}$ side lengths equal to $44 \AA$. The box length in $\mathrm{z}$ allowed to vary so as to maintain a constant pressure. The simulations used 5680 water molecules and the appropriate number of ions to give the intended molality. This system size gives a box size in the $\mathrm{z}$ direction equal to about $88 \AA$, half of which contains the salt solution. The molality of the solution is found from the average number of ions from $-z_{r}$ to $+z_{r}$ divided by the number of water molecules in the same region. All four ion pairs were simulated at a concentration of $1 \mathrm{~m}$ and an additional higher concentration. The solutions $\mathrm{NaCl}$ and $\mathrm{NaI}$ were simulated at $5.0 \mathrm{~m}, \mathrm{KCl}$ at $4.8 \mathrm{~m}$, the saturation concentration, and KI at $4.5 \mathrm{~m}$, the highest concentration for which an experimental value is available.[39] The $1 \mathrm{~m}$ simulations used 104 ions for the $\mathrm{NaCl}$ and $\mathrm{KCl}$ solutions, 102 ions for the KI solutions, and 100 ions for the NaI solutions. The $5.0 \mathrm{~m} \mathrm{NaCl}$ solution contained 496 ions, the $4.8 \mathrm{~m} \mathrm{KCl}$ solution contained 456 ions, the $5.0 \mathrm{~m} \mathrm{NaI}$ solution 434 ions, and the $4.5 \mathrm{~m} \mathrm{KI}$ solution contained 430 ions.

The potential model. The simulations used a recently developed model which includes both charge transfer and polarizability. [42, 43, 44] Charge transfer is treated by allowing charge to be transferred between pairs. The amount of charge transfer depends on the distance between pairs and the model has charges which change every time step, according to the local structure. The TIP4P-FQ+DCT model, which treats polarizability with the fluctuating charge method, is used for water.[42] Polarizability for the ions is treated using the Drude model, in which a charge site is placed on a spring. $[45,46]$ The interaction between two ions $i$ and $j$ is given by

$$
\begin{aligned}
E_{i j}= & 4 \epsilon_{i j}\left[\left(\frac{\sigma_{i j}}{r_{i 1 j 1}}\right)^{12}-\left(\frac{\sigma_{i j}}{r_{i 1 j 1}}\right)^{6}\right]+\sum_{\alpha \beta} \frac{q_{i \alpha} q_{j \beta}}{r_{i \alpha j \beta}} S_{i j}\left(r_{i \alpha j \beta}\right) \\
& +\left(-\mu_{i j}^{C T}\left|q_{i j}^{C T}\right|+\frac{1}{2} \eta_{i j}^{C T}\left(q_{i j}^{C T}\right)^{2}\right)
\end{aligned}
$$

with a Lennard-Jones interaction between the ion centers, Coulombic interactions between all sites on different ions, and a charge transfer energy term. The distance between sites $\alpha$ and $\beta$ on ions $i$ and $j$ is $r_{i \alpha j \beta}$, with the first site corresponding to 
the ion center and the second corresponding to the Drude position. The Coulombic interactions are damped at short-range according to

$$
S_{i j}\left(r_{i \alpha j \beta}\right)=1-\left(1+\frac{r_{i \alpha j \beta}}{2 a_{i j}}\right) e^{-r_{i \alpha j \beta} / a_{i j}}
$$

where $a_{i j}$ determines the amount of screening. The charge transfer between pairs is distance dependent as given by

$$
q_{i j}^{C T}= \begin{cases}Q_{i j}^{C T} & \text { if } r_{1 i j 1}<R_{1}^{C T}, \\ \frac{1}{2} Q_{i j}^{C T}\left[1+\cos \left(\pi \frac{r_{i 1 j 1}-R_{1}^{C T}}{R_{2}^{C T}-R_{1}^{C T}}\right)\right] & \text { if } R_{1}^{C T} \leq r_{i 1 j 1} \leq R_{2}^{C T} \\ 0 & \text { if } r_{i 1 j 1}>R_{2}^{C T},\end{cases}
$$

as characterized by a charge transfer amount, $Q_{i j}^{C T}$ and two distances, which reduces the charge transfer to zero over the range $R_{1}^{C T}$ to $R_{2}^{C T}$. The charge is transferred to the ion center and the Drude charge remains unchanged. No charge is transferred between like ions. The three charge transfer parameters are taken from quantum calculations and not treated as adjustable. The charge transfer for the ion pairs was chosen so that the amount of charge transfer reproduces the results for an ion in a crystal environment, with the ion surrounded by six nearest counter-ion neighbors. This gives a charge transfer amount for the $\mathrm{NaCl}$ of $0.017 \mathrm{e}$, which is in good agreement with a previous study which reported a charge for sodium atom in solid $\mathrm{NaCl}$ equal to $0.90 e$, and so a charge transfer of $0.0167 e$ from each six neighbors. [47] The cut-off distances $R_{1}^{C T}$ and $R_{2}^{C T}$ are chosen to match the length scales of charge transfer from the quantum calculations.

As in previous studies, [34, 21, 23] the usual Lorentz-Berthelot combining rule for $\sigma$ is modified by introducing a scaling factor, as given by

$$
\sigma_{i j}=\lambda_{i j} \frac{1}{2}\left(\sigma_{i}+\sigma_{j}\right)
$$

This modification is only used for unlike ion pairs, and Lorentz-Berthelot combining rule $\left(\epsilon_{i j}=\left(\epsilon_{i} \epsilon_{j}\right)^{1 / 2}\right)$ is used.

The parameters for the charge transfer model are given in Tables 1 and 2. Each ion pair has three parameters which are used for optimization. These are the LennardJones scaling parameter for $\sigma, \lambda_{i j}$, the Coulombic damping coefficient, $a_{i j}$, and $\mu_{i j}^{C T}$ from the charge transfer energy equation. The other charge transfer energy parameter, $\eta_{i j}^{C T}$, was kept fixed, since most properties were not found to be strongly dependent on this term. Only the ion-ion parameters are being optimized, the waterwater and water-ion parameters are taken from previous studies. [42, 43, 44] The three 
parameters were fit to reproduce the osmotic coefficient at different concentrations and the optimized ion dimer geometry. We followed a simple optimization procedure in which a value of $a_{i j}$ was chosen, then a $\lambda_{i j}$ value was found which gives the correct dimer geometry, then $\mu_{i j}^{C T}$ was varied to find optimal osmotic coefficients. Then a different value of $a_{i j}$ was chosen, until an overall best set of coefficients was found.

Simulation details. All simulations were run using our own molecular dynamics program. Simulations were carried out in the isothermal, isobaric ensemble at a temperature of $298 \mathrm{~K}$ and a pressure of 1 atm, using a Nosé-Hoover thermostat and an Anderson barostat.[48] The charge equilibration for the fluctuating charges is handled via an extended Lagrangian approach [49] using charge normal modes. [50, 42] The Drude variables are given a mass equal to $0.4 \mathrm{amu}$, which is subtracted from the mass of the ion center, and propagated as described by Lamoureux and Roux.[51] The simulations use a 1 fs time step and Ewald summation for the longrange electrostatics. [48] The osmotic pressure simulations were run for at least 14 nanoseconds at $1 \mathrm{~m}$ and 2 nanoseconds at the higher concentrations. Bonds were constrained using the SHAKE algorithm.[48] All bulk salt solution simulations used 2840 water molecules. The $1 \mathrm{~m}$ simulations used 104 and the $4.5 \mathrm{~m}$ simulations used 460 ions. Calculations of the potential of mean forces and the ion charges as a function of ion pair distance were done with umbrella sampling, with two ions and 2840 water molecules. These calculations used umbrella sampling with umbrella windows separated by $1 \AA$.

Quantum calculations. Quantum theory of atoms in molecules (QTAIM) [52] analysis is carried out for $\mathrm{NaCl}, \mathrm{KCl}, \mathrm{NaI}$, and $\mathrm{KI}$ dimers and clusters. The $\mathrm{NaCl}$ and $\mathrm{KCl}$ dimer studies use MP2 with the aug-cc-pvTZ basis set on $\mathrm{Na}$ and $\mathrm{Cl}$ and the $6-311++\mathrm{G}(2 \mathrm{~d}, 2 \mathrm{p})$ basis for $\mathrm{K}$. These studies used the QTAIM analysis software developed by Henkelmann and coworkers. [53] All other studies use the AIMAll software package.[54] Quantum calculations with chloride are carried out in NWChem version 5.1.[55] Calculations with iodide use Gaussian09.[56] For iodide, the effective core potential (ECP) MWB46 is used at the Hartree-Fock (HF) level.

To better understand CT in the condensed phase, clusters of ion pairs are studied. A face-centered cubic cell consisting of 27 ions placed at their distances in salt crystals is formed. A single-point calculation is carried out at HF level; due to computational expense, the basis set size was reduced to aug-cc-pvDZ for $\mathrm{Na}$ and $\mathrm{Cl}$. Periodic boundary conditions (PBC) are not applied, and the dielectric is $\varepsilon=1$. A calculation was attempted with $\varepsilon=6$, which is more relevant to the solid salt environment but proved to be too computationally demanding. In these "mini-crystal" clusters, the charge of the central ion is calculated. These calculations show that CT per ligand is about half of the CT seen for gas-phase dimers. Calculations on dimers with the dielectric equal to six also show $\mathrm{CT}$ half of that for dimers when the dielectric is one. 


\section{Results}

The optimized parameters for the ion pairs, along with previous parameters for the ion water interactions, are given in Table 2. The properties of the optimized models, compared to those used for parameterization, the dimer distance and the osmotic coefficients, are shown in Table 3. The osmotic coefficients for the four salts are shown in Figure 2. The osmotic coefficients of the model are in good agreement, showing both the dependence on ion pair identity and concentration.

Pair correlation functions between the ion-pairs, at concentrations of $1 \mathrm{~m}$ and $4.5 \mathrm{~m}$, show a range of structures (Figure 3). The ion pairs $\mathrm{NaCl}$ and $\mathrm{KCl}$ show a contact ion peak around $3 \AA$ as well as a solvent separated peak and a third smaller peak. These pair correlation functions are qualitatively similar to others calculated using non-polarizable potentials for $\mathrm{NaCl}[57,17,58,24,33,25,18,19,59]$ and $\operatorname{KCl}[57,25,18,19]$ including those with ion-ion interactions that have been optimized to reproduce osmotic pressures[21, 34] and activities. [37] The pair NaI has the smallest contact pair peak, with a bigger solvent separated peak, in agreement with previous simulations. [34, 37] A small contact pair peak for $\mathrm{NaI}$ is consistent with it having the largest osmotic pressure. KI has a large contact pair peak and no solvent separated peak. Potential of mean force calculations for KI find a very shallow free energy minimum for the solvent separated peak.[18] A larger contact pair peak for KI, and therefore a bigger amount of ion pairing, is consistent with KI having a small osmotic pressure.

The potentials of mean force, $\mathrm{w}(\mathrm{r})$, between ion pairs (from simulations with only the two ions) show similar results to the pair correlation functions (Figure 4) at higher concentrations. (Each $\mathrm{w}(\mathrm{r})$ is adjusted so that the value at a separation of 8 $\AA$ is equal to $-1 / 4 \pi \epsilon_{0} \epsilon$, where $\epsilon_{0}$ is the vacuum permittivity and $\epsilon$ is the dielectric constant of the model, 78.) KI has the deepest contact pair minimum and no solvent separated local minimum. NaI has a solvent separated minimum about as deep as that of the contact pair.

The number of contact pairs, $\mathrm{N}_{C P}$, can be found by integrating the ion pair correlation function, $\mathrm{g}(\mathrm{r})$, over the contact pair peak, as given by

$$
N_{C P}=4 \pi \rho_{p} \int_{0}^{r_{c u t}} g(r) r^{2} d r
$$

where $\rho_{p}$ is the number density of ion pairs. [58] This corresponds to integrating out to a cut-off distance of $3.5 \AA$ for $\mathrm{NaCl}, 4.0 \AA$ for $\mathrm{KCl}$ and $\mathrm{NaI}$, and $4.5 \AA$ for KI. Note that there is an arbitrariness of this definition of an ion pair, given that the pair correlation functions are all very close to one at the cut-off distances, but these cut-off distances are reasonable given that they are $1.2 \AA$ to $1.5 \AA$ (or about half an ionic radius) larger than the dimer energy minimum distance (Table 3 ). The values are given in Table 4. At $1 \mathrm{~m}, \mathrm{NaCl}, \mathrm{KCl}$ and $\mathrm{NaI}$ all show about the same 
amount of ion pairing, from 0.1 to 0.2 , which means 10 to $20 \%$ of the time an ion is paired. KI shows increased pairing, so that an ion pair is formed about $80 \%$ of the time. At higher concentrations, there is more ion pairing, but the increase is not as much as the ratio of the concentrations. This means there is relatively less ion pairing, compared to random chance, as indicated by the contact pair peaks in the pair correlation functions which decrease with concentration. Other studies, for $\mathrm{NaCl}$ and $\mathrm{KCl}$ solutions, have also seen a decrease in the contact pair with increased concentration. $[25,21]$ These studies find ion pairing fairly close to the CT model results, with $N_{C P}$ less than 0.5 and smaller for $\mathrm{NaCl}$ than $\mathrm{KCl}$, but the results do depend on the water and ion models. [25, 21, 17]

The random probability to have two ions together can be found from Equation 6 by setting $\mathrm{g}(\mathrm{r})$ to 1 at all distances beyond an excluded volume radius, $\mathrm{r}_{e v}$. This gives $N_{C P}^{r a n d o m}=(4 \pi / 3) \rho_{p}\left(r_{c u t}^{3}-r_{e v}^{3}\right)$. Values of $\mathrm{r}_{e v}$ can be found from the pair correlation functions, at the distance where the $\mathrm{g}(\mathrm{r})$ first equals 1 . This gives values of $\mathrm{r}_{e v}$ equal to $2.45 \AA, 2.89 \AA, 3.00 \AA$, and $3.18 \AA$, for $\mathrm{NaCl}, \mathrm{KCl}, \mathrm{NaI}$, and KI, respectively. The amount of contact pairing just by random chance, at $1 \mathrm{~m}$, is then $0.073,0.102,0.092$, and 0.144 for $\mathrm{NaCl}, \mathrm{KCl}, \mathrm{NaI}$, and $\mathrm{KI}$, respectively. For $\mathrm{NaCl}$ and $\mathrm{KCl}$, the amount of ion pairing is about a factor of two higher than the random value and for NaI, it is only slightly higher. At $4.5 \mathrm{~m}, N_{C P}^{\text {random }}$ will be 4.5 times the value at $1 \mathrm{~m}$, giving 0.33 , $0.46,0.41$, and 0.65 for $\mathrm{NaCl}, \mathrm{KCl}, \mathrm{NaI}$, and $\mathrm{KI}$, respectively. At this concentration, only KI has an ion pairing significantly higher than random.

The relationship between ion pairing and osmotic pressure is checked by changing the value of $\mu_{i j}^{C T}$ which is part of the charge transfer energy term. This term is shortranged and can be used to change the amount of ion pairing while leaving the rest of the interactions unchanged. The ion pairing for the optimized models and a model with an increased value of $\mu_{i j}^{C T}$ (and so more ion pairing) are shown in Figure 5. All models show a decrease in the osmotic coefficient with increased contact pairing, in agreement with previous studies. [21, 23] The three ion pairs $\mathrm{NaCl}, \mathrm{KCl}$, and $\mathrm{NaI}$ all show a similar relationship between contact pairing and osmotic coefficient, $\phi$, with KI showing different dependence. The other ions pairs with the large amount of contact pairing seen in KI would be expected to have a much smaller $\phi$ than that of KI. One noticeable difference between KI and the others from Figure 3 is the lack of solvent separated pair. Including the solvent separated pair in the definition of an ion pair would lead to relatively more pairing for $\mathrm{NaCl}, \mathrm{KCl}$, and $\mathrm{NaI}$ (Table 4). This definition of ion pairing, labelled $N_{C P+S S P}$, is found by integrating the $\mathrm{g}(\mathrm{r})$ to $6 \AA$. Even with this definition of ion pairing, KI has more pairing than $\mathrm{KCl}$ at $1 \mathrm{~m}$, although not by that much, but has a larger osmotic coefficient, implying that more than ion pairing is important. Note that at the higher concentration, $N_{C P+S S P}$ is larger for $\mathrm{KCl}$ than for $\mathrm{KI}$.

Additional characterization can be done by examining the ion clusters formed in the solutions. Ions are taken to be paired if they are within the cut-off distance, $\mathrm{r}_{c u t}$, given above, and are grouped into larger clusters if any ion is part of more than one 
pair. [30, 32] The cluster distributions are given by $\mathrm{p}(\mathrm{n})$, which is the average number of clusters of size n divided by the total number of ions, $N_{\text {ions }}$. This definition of $\mathrm{p}(\mathrm{n})$ is normalized so that the sum over all cluster sizes times the number of ions in that cluster equals 1 , or

$$
\sum_{n=1}^{N_{\text {ion }}} p(n) n=1
$$

(For example, if only single ions are present, there would be $N_{\text {ions }}$ a particle clusters and $\mathrm{p}(1)=1$. If all ions are paired in two particle clusters, there would be $N_{\text {ions }} / 22$ particle clusters and $\mathrm{p}(2)=1 / 2$.) The cluster distributions are shown in Figures 6(a) and $6(\mathrm{~b})$ and the values for the smaller clusters are given in Table 3. The $\mathrm{NaCl}$, $\mathrm{KCl}$, and $\mathrm{NaI}$ solutions show similar clustering with about 80 to $90 \%$ of the ions are unpaired at $1 \mathrm{~m}$. For the KI solution only about half the ions are unpaired at $1 \mathrm{~m}$. A significant fraction of the ions are present as pairs (the values of $\mathrm{p}(2)$ are numerically different from $\mathrm{N}_{C P}$ because there is a different normalization and also $\mathrm{N}_{C P}$ includes ion pairs that are present as part of larger clusters). Larger clusters are also formed. At $1 \mathrm{~m}$, for $\mathrm{NaCl}$ no clusters larger than 7 are observed and for $\mathrm{NaCl}, \mathrm{KCl}$, and $\mathrm{NaI}$ $\mathrm{p}(\mathrm{n})$ appears to drop off exponentially. Similar results for $\mathrm{NaCl}$ and $\mathrm{KCl}$ have been reported by Chen and Pappu.[30] Much larger clusters are observed for KI. At 1m, clusters up to size 27 are seen and at $4.5 \mathrm{~m}$, there are clusters of around 300 ions, which represents about $2 / 3$ of the total ions of the system. Visual inspection of these large clusters reveals that they are extended throughout the solution and do not represent compact crystal-like structures. These clusters are probably driven by a strong tendency for KI to form pairs, which can then form chains. The amount of clustering will depend on the cut-off used for pairing, $\mathrm{r}_{c u t}$, but even if this value is reduced to $4.0 \AA$ (the value used for $\mathrm{KCl}$ and $\mathrm{NaI}$ ) these large clusters are still found.

The clusters can be characterized in terms of composition and structure. The radius of gyration, $\mathrm{R}_{G}$, of the clusters as a function of cluster size is shown in Figure 7 . Shown are the results for $\mathrm{NaCl}$ and $\mathrm{KI}$ at a concentration of $4.5 \mathrm{~m}$. The results for $\mathrm{KCl}$ and $\mathrm{NaI}$ are very similar to those of $\mathrm{NaCl}$ and the results at the lower concentration are similar as well, except larger clusters are not formed. For comparison, the radius of gyration for clusters with a linear geometry and in an $\mathrm{NaCl}$ lattice geometry are plotted. For the linear clusters, $\mathrm{R}_{G}$ increases linearly with cluster size $\mathrm{n}$ and for the cubic geometry, $\mathrm{R}_{G}$ increases as $\mathrm{n}^{1 / 3}$. When plotted on a log-log scale, the slopes will give the exponent of the dependence on $\mathrm{n}$. The lines are generated using a nearest neighbor distance of $3.5 \AA$, appropriate for KI. Up to about a cluster size of 10 , the $\mathrm{R}_{G}$ increases as a linear chain. For the larger KI clusters, $\mathrm{R}_{G}$ does not increase linearly, but the clusters are still appreciably larger than an ideal (compact) crystal would be.

The composition of the clusters can be described using its formal charge, which 
gives the relative number of cations to anions. The charge of the clusters in the 4.5 $\mathrm{m}$ solution as a function of size is given in Figure 8. The cluster charges at $1 \mathrm{~m}$ are similar. Overall, clusters larger than two are positively charged, on average. Of the ions not part of a cluster, there tends to be small preference for anions (since the larger clusters have a preference for cations). This is an excess of anions about 0.1 to $1 \%$ at $1 \mathrm{~m}$ and about 2 to $8 \%$ at $4.5 \mathrm{~m}$, with KI (having more larger clusters) at the high end.

The charge of an ion as a function of distance between the solvated cation and anion is shown in Figure 9. This is for a solution with a single ion pair. The anions get more negatively charged when an ion pair is formed, because there is more charge transfer from the anion to water than there is to a cation (see Table 2). The anion charge is slightly larger around a separation of $4.5 \AA$, about a half Angstrom greater than the minimum between the contact and solvent separated pairs (see Figure 4). At this separation, some solvation shell water molecules are lost, resulting in less charge transfer. A more negative chloride ion upon pairing with sodium is seen in analysis of classical potentials using ab initio calculations. [47] The charge of the cation changes less upon pairing, because the difference in the cation-anion and cation-water charge transfer amounts are not as large. Similar results for LiF ion pair formation from $a b$ initio molecular dynamics have been reported, in which the anion charge changes more than the cation charge as a pair is formed.[60]

There is more charge transferred to a water molecule from an anion than from a water molecule to a cation. This results in a net negative charge on the water molecules.[44, 47] The average charges of the ions and water are shown on Table 4. An ion pair transfers about $-0.1 e$ of a charge to water, on average among these ion types. That charge is shared among the water molecules of the system, probably mostly on the first solvation shell,[44, 47] and gets larger, per water molecule, as the ion concentration increases. The charge of the water, as a result of charge transfer from an ion, has been shown to have an important influence on water translational diffusion time scales.[61]

\section{Conclusions}

Potentials for ions and water which include polarizability and charge transfer have been previously demonstrated to give accurate single ion properties, including hydration free energies and solvent structure.[43, 44] By a combination of quantum calculations of ion/water clusters, to determine the amount of charge transfer between ion pairs in solution, and modification of the Lorentz-Berthelot combining rules for the Lennard-Jones interaction, the models can accurately reproduce the osmotic coefficient for concentrated salt solutions (Figure 2), as has been demonstrated for other potential models. $[17,33,34,21,23]$ Because the osmotic pressure is sensitive to the amount of ion paring[21, 23] (and see Figure 5), these optimized potentials can be used to examine the amount of ion pairing and larger ion cluster formation. 
Among the four solutions, $\mathrm{NaCl}, \mathrm{KCl}$, and $\mathrm{NaI}$ are fairly similar, with contact pair and a solvent separated peaks in the ion-ion pair correlation functions (Figure 3) and, while the contact pair peaks may appear large, the amount of ion pairing is not that significant. At a concentration of $1 \mathrm{~m}, 10-20 \%$ of ions are paired; the amount of pairing purely by chance would be about $10 \%$. At a higher concentration of $4.5 \mathrm{~m}$, none of these three pairs demonstrates pairing much larger than random. Clusters larger than pairs are observed, with probability that falls off exponentially with size (Figures 6(a) and 6(b)). The KI solution behaves much differently. There is substantially more ion pairing and clustering, so that even at $1 \mathrm{~m}$ most ions are paired or in larger clusters. The clusters for all four ion types form extended almost linear structures, with no evidence of crystal-type compact structures (Figure 7).

The cations tend to cluster more than the anions, so that clusters of size bigger than two tend to be positively charged (Figure 8). This is true for even numbered clusters, as well, which might be expected to have an identical number of anions and cations. A preference for cations in the clusters was seen in previous simulations of $\mathrm{NaCl}$ and other alkali halide salts. [30, 32] $\mathrm{KI}$ and $\mathrm{KCl}$ form larger clusters and also tend to have clusters with a smaller anion/cation imbalance. NaI tends to pair and cluster the least, which is consistent with the law of matching affinities, since this pair is the most different in terms of hydration free energies among these four ion pairs (KI is the most similar). We find that the smaller cation forms fewer clusters, in disagreement with previous simulation results using non-polarizable potentials[30, 18] indicating that the clustering depends on the details of the potential models, as has been noted.[18] Our results are in agreement with the 2D IR experiments of Bian, et al., [27] (with $\mathrm{Li}^{+}, \mathrm{Na}^{+}, \mathrm{K}^{+}$, and $\mathrm{Cs}^{+}$as the cation and $\mathrm{SCN}^{-}$as the anion). The osmotic pressure-optimized (non-polarizable) potentials of Luo and Roux find more ion pairing for $\mathrm{KCl}$ than $\mathrm{NaCl}$, in agreement with our results.[21] For any of these anions in our study or the others $\left(\mathrm{Cl}^{-}, \mathrm{Br}^{-}, \mathrm{I}^{-}\right.$, and $\left.\mathrm{SCN}^{-}\right)$, the larger cation would match the water affinity the best, so the larger cations should cluster more. It is surprising that the cations have a stronger tendency to be part of a cluster, since the ions $\mathrm{K}^{+}$and $\mathrm{Na}^{+}$are more hydrophilic than $\mathrm{Cl}^{-}$and $\mathrm{I}^{-}$, with larger hydration free energies.[40] This may be because these particular anions are larger than the cations and excess cations can be arranged around the larger anions without being in as close contact as two large anions would be around a cation. Size matching has been proposed to be important for ion pairing, [62] and size matching may be a factor for the larger clusters as well.

\section{Acknowledgements}

This work was supported by the National Science Foundation under contract number CHE-0611679. This material is based upon work supported by the Louisiana Optical Network Institute (LONI) and by the National Science Foundation under the NSF EPSCoR Cooperative Agreement No. EPS-1003897, with additional support 
from the Louisiana Board of Regents. MS gratefully acknowledges support from the State of Louisiana Board of Regents.

\section{References}

\section{References}

[1] Y. Wang, P. Nordlander, N. H. Tolk, Extended Huckel theory for ionic molecules and solids: An application to alkali halides, J. Chem. Phys. 89 (1988) 4163.

[2] J. Kim, H. M. Lee, S. B. Suh, D. Majumdar, K. S. Kim, Comparative ab initio study of hte structures, energetics an spectra of $\mathrm{X}^{-} \cdot\left(\mathrm{H}_{2} \mathrm{O}\right)_{n=1-4}[\mathrm{X}=\mathrm{F}, \mathrm{Cl}, \mathrm{Br}$, I] clusters, J. Chem. Phys. 113 (13) (2000) 5259-5272.

[3] W. Klopper, J. van Duijnevedt-van de Rijdt, R. B. van Duijneveldt, Computational determinination of equilibrium geometry and dissociation energy of the water dimer, Phys. Chem. Chem. Phys. 2 (2000) 2227-2234.

[4] W. M. Haynes (Ed.), CRC Handbook of Chemistry and Physics, CRC Press, Internet Version, 92nd edn., 2012.

[5] R. M. Fuoss, Conductimetric determination of thermodynamic pairing constants for symmetrical electrolytes, Proc. Natl. Acad. Sci. USA 77 (1980) 34-38.

[6] C. B. Chan, N. H. Tioh, G. T. Hefter, Flouride complexes of the alkali metal ions, Polyhedron 3 (1984) 845-851.

[7] U. Kaatze, T. O. Hushcha, F. Eggers, Ultrasonic broadband spectroscopy of liquids a research tool in pure and applied chemistry and chemical physics, J. Solution Chem. 29 (2000) 299-368.

[8] E. A. Aziz, A. Zimina, M. Freiwald, S. Eisenbit, W. Eberhardt, Molecular and electronic structure in $\mathrm{NaCl}$ electrolytes of varying concentration: Identification of spectral fingerprints, J. Chem. Phys. 124 (2006) 114502.

[9] W. Wachter, S. Fernandez, R. Buchner, G. Hefter, Ion association and hydration in aqueous solutions of $\mathrm{LiCl}$ and $\mathrm{Li} 2 \mathrm{SO} 4$ by dielectric spectroscopy, J. Phys. Chem. B 111 (2007) 9010-9107.

[10] F. Sebe, K. Nishikawa, Y. Koga, Spectrum of excess partial molar absorptivity. Part II: a near infrared spectroscopic study of aqueous Na-halides, Phys. Chem. Chem. Phys. 14 (2012) 4433-4439.

[11] Y. Marcus, G. Hefter, Ion Pairing, Chem. Rev. (2006) 4585-461. 
[12] M. Berkowitz, O. A. Karim, J. A. McCammon, Sodium chloride ion pair interaction in water: computer simulation, Chem. Phys. Lett. 105 (1984) 577.

[13] B. M. Pettitt, P. J. Rossky, Alkali halides in water: ion-solvent correlations and ion- ion potentials of mean force at infinite dilution, J. Chem. Phys. 84 (1986) 5836.

[14] D. E. Smith, L. X. Dang, Computer simulations of $\mathrm{NaCl}$ association in polarizable water, J. Chem. Phys. 100 (1994) 3757-3766.

[15] J. Van Eerden, W. J. Briels, S. Harkema, D. Feil, Potential of mean force by thermodynamic integration: molecular-dynamics simulation of decomplexation, Chem. Phys. Lett. 164 (1989) 370-376.

[16] L. R. Pratt, G. Hummer, A. E. Garcia, Ion pair potentials-of-mean-force in water, Biophysical Chem. 51 (1994) 147-165.

[17] B. Hess, C. Holm, N. van der Vegt, Osmotic coefficients of atomistic NaCl (aq) force fields, J. Chem. Phys. 124 (2006) 164509.

[18] C. J. Fennell, A. Bizjak, V. Vlachy, K. A. Dill, Ion Pairing in Molecular Simulations of Aqueous Alkali Halide Solutions, J. Phys. Chem. B 113 (2009) 67826791.

[19] C. J. Fennell, A. Bizjak, V. Vlachy, K. A. Dill, S. Sarupria, S. Rajamani, S. Garde, Ion Pairing in Molecular Simulations of Aqueous Alkali Halide Solutions, J. Phys. Chem. 113 (2009) 14837-14838.

[20] J. Timko, D. Bucher, S. Kayucak, Dissociation of $\mathrm{NaCl}$ in water from ab initio molecular dynamics simulations., J. Chem. Phys. 132 (2010) 114510.

[21] Y. Luo, B. Roux, Simulations of osmotic pressure in concentrated aqueous salt solutions, J. Phys. Chem. Lett. 1 (2010) 183-189.

[22] M. K. Ghosh, S. Re, M. Feig, Y. Sugita, C. H. Choi, Interionic hydration structures of $\mathrm{NaCl}$ in aqueous solution: a combined study of quantum mechanical cluster and QM/EFP-MD simulations, J. Phys. Chem. B 117 (2013) 289-295.

[23] Y. Luo, W. Jiang, H. Yu, A. D. MacKerell, B. Roux, Simulation study of ion pairing in concentrated aqueous salt solutions with a polarizable force field, Faraday Discuss. 160 (2013) 135-149.

[24] S. Bouaziz, S. Nasr, N. Jaâdane, M. C. Bellissent-Funnel, Local Order in Aqueous $\mathrm{NaCl}$ Solutions and Pure Water: X-ray Scattering and Molecular Dynamics Simulations Study, J. Phys. Chem. B 110 (2006) 23515-23523. 
[25] R. Mancinelli, A. Botti, M. A. Ricci, A. K. Soper, Hydration of Sodium, Potassium, and Chloride Ions in Solution and the Concept of Structure Maker/Breaker, J. Phys. Chem. B 111 (2007) 13570-13577.

[26] M. K. Cerreta, K. A. Berglund, The structure of aqueous solutions of some dihyrogen orthophosphates by laser Raman spectroscopy, J. Crystal Growth 84 (1987) 577-588.

[27] H. Bian, X. Wen, J. Li, H. Chen, S. Han, X. Sun, J. Song, W. Zhuang, J. Zheng, Ion clustering in aqueous solutions probed with vibrational energy transfer, Proc. Natl. Acad. Sci. USA 108 (2011) 4737-4742.

[28] L. Degrève, F. L. B. da Silva, Detailed microscopic study of $1 \mathrm{M}$ aqueous $\mathrm{NaCl}$ solution by computer simulations, J. Mol. Liq. 87 (2000) 217-232.

[29] D. M. Sherman, M. D. Collings, Ion association in concentrated $\mathrm{NaCl}$ brines from ambient to supercritical conditions: results from classical molecular dynamics simulations, Geochem. Trans. 3 (2002) 102-107.

[30] A. A. Chen, R. V. Pappu, Quantitative characterization of ion pairing and cluster formation in strong 1:1 electrolytes, J. Phys. Chem. B 111 (2007) 6469-6478.

[31] S. A. Hassan, Morphology of ion clusters in aqueous electrolytes, Phys. Rev. E 77 (2008) 031501.

[32] S. A. Hassan, Computer simulation of ion cluster speciation in concentrated aqueous solutions at ambient conditions, J. Phys. Chem. B 112 (2008) 1057310584.

[33] I. Kalcher, J. Dzubiella, Structure-thermodynamics relation of electrolyte solutions, J. Chem. Phys. 130 (2009) 134507.

[34] M. Fyta, I. Kalcher, J. Dzubiella, L. Vrbka, R. R. Netz, Ionic force field optimization based on single-ion and ion-pair solvation properties, J. Chem. Phys. 132 (2010) 024911.

[35] I. S. Joung, T. E. Cheatham III, Molecular dynamics simulations of the dynamic and energetic properties of alkali and halide ions using water-model-specific ion parameters, J. Phys. Chem. B 113 (2009) 13279-13290.

[36] B. Hess, C. Holm, N. van der Vegt, Cation specific binding with protein surface charges, Proc. Natl. Acad. Sci. USA 106 (2009) 13296-13300.

[37] M. Fyta, R. R. Netz, Ionic force field optimization based on single-ion and ionpair solvation properties: Going beyond standard mixing rules, J. Chem. Phys. 136 (2012) 124103. 
[38] K. D. Collins, Charge density-dependent strength of hydration and biological structure, Biophysical J. 72 (1997) 65-76.

[39] W. J. Hamer, Y. C. Wu, Osmotic coefficients and mean activity coefficients of uni-univalent electrolytes in water at 25 C, J. Phys. Chem. Ref. Data 1 (1972) $1047-1100$.

[40] M. D. Tissandier, K. A. Cowen, W. Y. Feng, E. Gundlach, M. H. Cohen, A. D. Earhart, J. V. Coe, T. R. Tuttle, Jr., The proton's absolute aqueous enthalpy and Gibbs free energy of solvation from cluster-on solvation data., J. Phys. Chem. A 102 (1998) 7787-7794.

[41] Y. Marcus, Thermodynamics of solvation of ions. Part 5.-Gibbs free energy of hydration at 298.15 K, J. Chem. Soc., Faraday Trans. 87 (1991) 2995-2999.

[42] A. J. Lee, S. W. Rick, The effects of charge transfer on the properties of liquid water, J. Chem. Phys. 134 (2011) 184507.

[43] M. Soniat, S. W. Rick, The effects of charge transfer on the aqueous solvation of ions, J. Chem. Phys. 137 (2012) 044511.

[44] M. Soniat, S. W. Rick, Charge transfer effects of ions at the liquid water/vapor interface, J. Chem. Phys. 140 (2014) 184703.

[45] S. J. Stuart, B. J. Berne, Effects of polarizability on the hydration of the chloride ion, J. Phys. Chem. 100 (1996) 11934-11943.

[46] H. Yu, T. W. Whitfield, E. Harder, G. Lamoureux, V. M. Anisimov, A. D. MacKerell, Jr., B. Roux, Simulating monovalent and divalent ions in aqueous solution using a Drude polarizable force field, J. Chem. Theory Comput. 6 (2010) $774-786$.

[47] B. Sellner, M. Valiev, S. M. Kathmann, Charge and electric field fluctuations in aqueous NaCl electrolyte, J. Phys. Chem. B 117 (2013) 10869-10882.

[48] M. P. Allen, D. J. Tildesley, Computer Simulation of Liquids, Oxford Univerity, 1987.

[49] S. W. Rick, S. J. Stuart, B. J. Berne, Dynamical fluctuating charge force fields: Application to liquid water, J. Chem. Phys. 101 (7) (1994) 6141-6156.

[50] L. R. Olano, S. W. Rick, Fluctuating charge normal modes: an algorithm for implementing molecular dynamics simulations with polarizable potentials, J. Comput. Chem. 26 (2005) 699-707. 
[51] G. Lamoureux, B. Roux, Modelling induced polarization with classical Drude oscillators: theory and molecular dynamics simulation algorithm, J. Chem. Phys. 119 (6) (2003) 3025-3039.

[52] R. F. W. Bader, Atoms in molecules, Acc. Chem. Res. 18 (1) (1985) 9-15.

[53] W. Tang, E. Sanville, G. Henkelman, A grid-based Bader analysis algorithm without lattice bias., J. Phys.: Condens. Matter 21 (2009) 084204.

[54] T. G. S. Todd A. Keith, AIMAll (Version 14.06.21), Overland Park KS, USA, (aim.tkgristmill.com), 2014.

[55] M. Valiev, E. J. Bylaska, N. Govind, K. Kowalski, T. P. Straatsma, H. J. J. van Dam, D. Wang, J. Nieplocha, E. Apra, T. L. Windus, W. A. de Jong, NWChem: a comprehensive and scalable open-source solution for large scale molecular simulations, Comp. Phys. Comm. 181 (2010) 1477.

[56] M. J. Frisch, G. W. Trucks, H. B. Schlegel, G. E. Scuseria, M. A. Robb, J. R. Cheeseman, G. Scalmani, V. Barone, B. Mennucci, G. A. Petersson, H. Nakatsuji, M. Caricato, X. Li, H. P. Hratchian, A. F. Izmaylov, J. Bloino, G. Zheng, J. L. Sonnenberg, M. Hada, M. Ehara, K. Toyota, R. Fukuda, J. Hasegawa, M. Ishida, T. Nakajima, Y. Honda, O. Kitao, H. Nakai, T. Vreven, J. A. Montgomery, Jr., J. E. Peralta, F. Ogliaro, M. Bearpark, J. J. Heyd, E. Brothers, K. N. Kudin, V. N. Staroverov, R. Kobayashi, J. Normand, K. Raghavachari, A. Rendell, J. C. Burant, S. S. Iyengar, J. Tomasi, M. Cossi, N. Rega, J. M. Millam, M. Klene, J. E. Knox, J. B. Cross, V. Bakken, C. Adamo, J. Jaramillo, R. Gomperts, R. E. Stratmann, O. Yazyev, A. J. Austin, R. Cammi, C. Pomelli, J. W. Ochterski, R. L. Martin, K. Morokuma, V. G. Zakrzewski, G. A. Voth, P. Salvador, J. J. Dannenberg, S. Dapprich, A. D. Daniels, Ö. Farkas, J. B. Foresman, J. V. Ortiz, J. Cioslowski, D. J. Fox, Gaussian 09 Revision A.1, Gaussian Inc. Wallingford CT, 2009.

[57] S. Chowdhuri, A. Chandra, Molecular dynamics simulations of aqueous $\mathrm{NaCl}$ and $\mathrm{KCl}$ solutions: Effects of ion concentrations on the single-particle, pair, and collective properties of ions and water molecules, J. Chem. Phys. 115 (2001) 3732-3741.

[58] G. V. Bondarenko, Y. E. Gorbaty, A. V. Okhulkov, A. G. Kalinichev, Structure and hydrogen bonding in liquid and supercritical aqueous $\mathrm{NaCl}$ solutions at a pressure of 1000 bar and temperatures up to $500 \mathrm{C}$ : A comprehensive experimental and computational study, J. Phys. Chem. A 110 (2006) 4042-4052.

[59] R. C. DeMille, V. Molinero, Coarse-grainsed ions without charges: reproducing the solvation structure of $\mathrm{NaCl}$ in water using short-ranged potentials, J. Chem. Phys. 131 (2009) 034107. 
[60] E. Pluhar̆ová, O. Marsalak, B. Schmidt, P. Jungwirth, Ab initio molecular dynamics approach to a quantative description of ion pairing in water, J. Phys. Chem. Lett. 4 (2013) 4177-4181.

[61] Y. Yao, Y. Kanai, M. L. Berkowitz, Role of charge transfer in water diffusivity in aqueous ionic solutions, J. Phys. Chem. Lett. 5 (2014) 2711-2716.

[62] M. Lund, B. Jagado-Cwkiklik, C. E. Woodward, R. Vácha, P. Jungwirth, Dielectric interpretation of specificity of ion pairing in water, Phys. Chem. Lett. 1 (2009) 300-303. 
Table 1 The Lennard-Jones well-depth, $\epsilon$, and radius, $\sigma$, the Drude charge and polarizability. [43, 44]

\begin{tabular}{lcccc}
\hline \hline & $\begin{array}{c}\epsilon \\
(\mathrm{kcal} / \mathrm{mol})\end{array}$ & $\begin{array}{c}\sigma \\
(\AA)\end{array}$ & $\begin{array}{c}\mathrm{q}_{D} \\
(\mathrm{e})\end{array}$ & $\begin{array}{c}\alpha \\
\left(\AA^{3}\right)\end{array}$ \\
\hline $\mathrm{Na}^{+}$ & 0.0407 & 2.320 & -0.687597 & 0.157 \\
$\mathrm{~K}^{+}$ & 0.0497 & 3.030 & -1.580968 & 0.830 \\
$\mathrm{Cl}^{-}$ & 0.1490 & 3.720 & -4.062989 & 5.482 \\
$\mathrm{I}^{-}$ & 0.1854 & 4.695 & -5.562578 & 10.272 \\
\hline \hline
\end{tabular}

Table 2 Charge transfer, electrostatic damping, and Lennard-Jones combining rule parameters for ion pairs and ion-water.[43, 44]

\begin{tabular}{lccccccc}
\hline \hline & $\begin{array}{c}\mathrm{Q}_{C T} \\
(\mathrm{e})\end{array}$ & $\begin{array}{c}\mathrm{r}_{C T 1} \\
(\AA)\end{array}$ & $\begin{array}{c}\mathrm{r}_{C T 2} \\
(\AA)\end{array}$ & $\begin{array}{c}\mu_{C T} \\
(\mathrm{kcal} / \mathrm{mol} / \mathrm{e})\end{array}$ & $\begin{array}{c}\eta_{C T} \\
\left(\mathrm{kcal} / \mathrm{mol} / \mathrm{e}^{2}\right)\end{array}$ & $\begin{array}{c}\mathrm{a} \\
(\AA)\end{array}$ & $\lambda$ \\
\hline $\mathrm{Na}^{+}-\mathrm{Cl}^{-}$ & 0.022 & 1.8 & 5.5 & 381.73 & 232.35 & 0.64 & 1.036 \\
$\mathrm{~K}^{+}-\mathrm{Cl}^{-}$ & 0.024 & 2.0 & 5.5 & 332.99 & 232.35 & 0.64 & 1.065 \\
$\mathrm{Na}^{+}-\mathrm{I}^{-}$ & 0.024 & 2.2 & 5.5 & 341.12 & 232.35 & 0.69 & 1.030 \\
$\mathrm{~K}^{+}-\mathrm{I}^{-}$ & 0.036 & 2.3 & 5.6 & 324.88 & 232.35 & 0.70 & 1.035 \\
$\mathrm{Na}^{+}-\mathrm{H}_{2} \mathrm{O}$ & 0.033 & 1.7 & 3.3 & 275.33 & 1602.6 & 0.10 & 1.0 \\
$\mathrm{~K}^{+}-\mathrm{H}_{2} \mathrm{O}$ & 0.024 & 2.0 & 3.7 & 304.57 & 6306.7 & 0.10 & 1.0 \\
$\mathrm{Cl}^{-}-\mathrm{H}_{2} \mathrm{O}$ & 0.057 & 1.9 & 3.1 & 95.51 & 995.8 & 0.60 & 1.0 \\
$\mathrm{I}^{-}-\mathrm{H}_{2} \mathrm{O}$ & 0.075 & 2.0 & 3.6 & 26.80 & -896.2 & 0.66 & 1.0 \\
\hline \hline
\end{tabular}

Table 3 Properties used in potential refinement: the optimized dimer distance, compared to the quantum mechanical results and the osmotic coefficients at different concentrations, compared to experiment.[39]

\begin{tabular}{lcccccccc}
\hline \hline & \multicolumn{2}{c}{ dimer $\mathrm{r}_{\text {min }}(\AA)$} & \multicolumn{3}{c}{ osmotic coefficient } & \multicolumn{3}{c}{ osmotic coefficient } \\
& model & $\mathrm{QM}$ & $\mathrm{c}(\mathrm{m})$ & model & experiment & $\mathrm{c}(\mathrm{m})$ & model & experiment \\
\hline $\mathrm{NaCl}$ & 2.35 & 2.30 & 1.0 & $0.95 \pm 0.04$ & 0.936 & 5.0 & $1.21 \pm 0.05$ & 1.191 \\
$\mathrm{KCl}$ & 2.67 & 2.67 & 1.0 & $0.90 \pm 0.04$ & 0.898 & 4.8 & $0.98 \pm 0.05$ & 0.990 \\
$\mathrm{NaI}$ & 2.68 & 2.68 & 1.0 & $1.01 \pm 0.04$ & 0.989 & 5.0 & $1.44 \pm 0.05$ & 1.415 \\
$\mathrm{KI}$ & 3.00 & 3.10 & 1.0 & $0.93 \pm 0.04$ & 0.928 & 4.5 & $1.05 \pm 0.04$ & 1.033 \\
\hline \hline
\end{tabular}




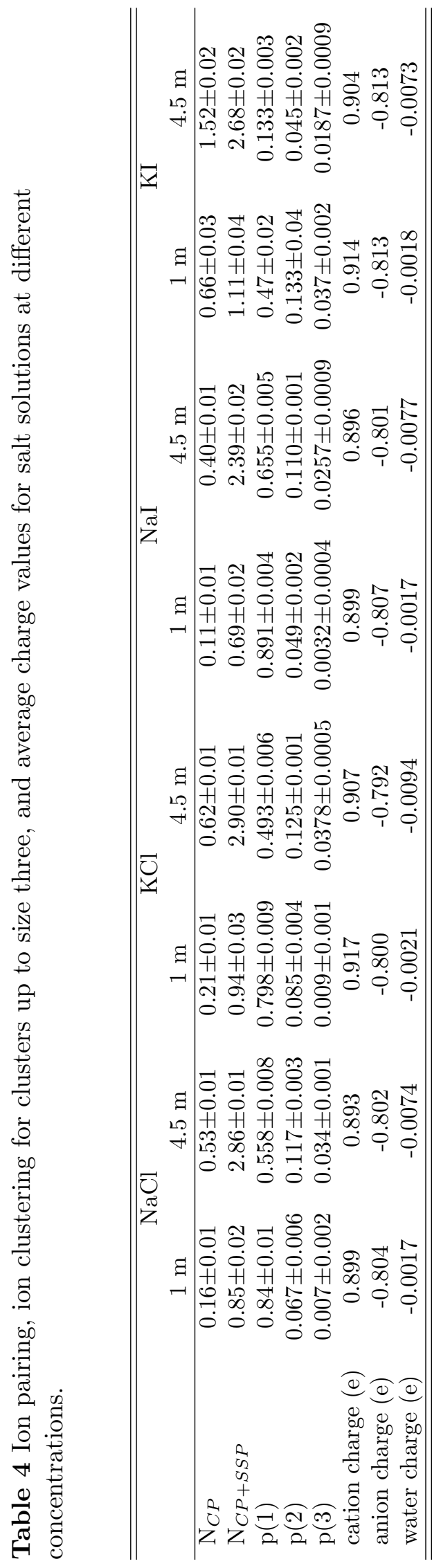




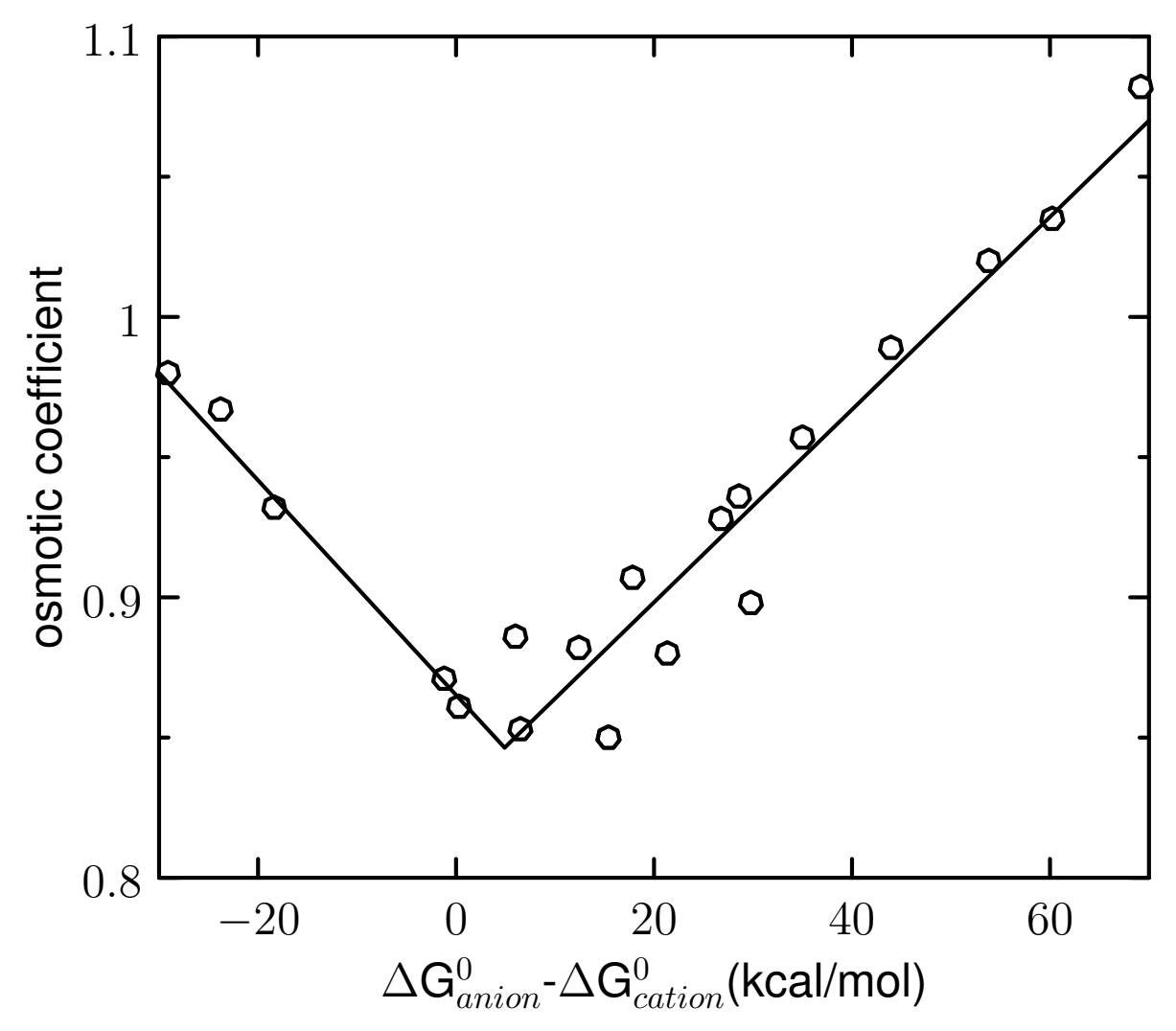

Figure 1 The difference in the absolute single-ion solvation free energy of the anion and the cation and the osmotic coefficient at $1 \mathrm{~m}$ for alkali halide salts. 

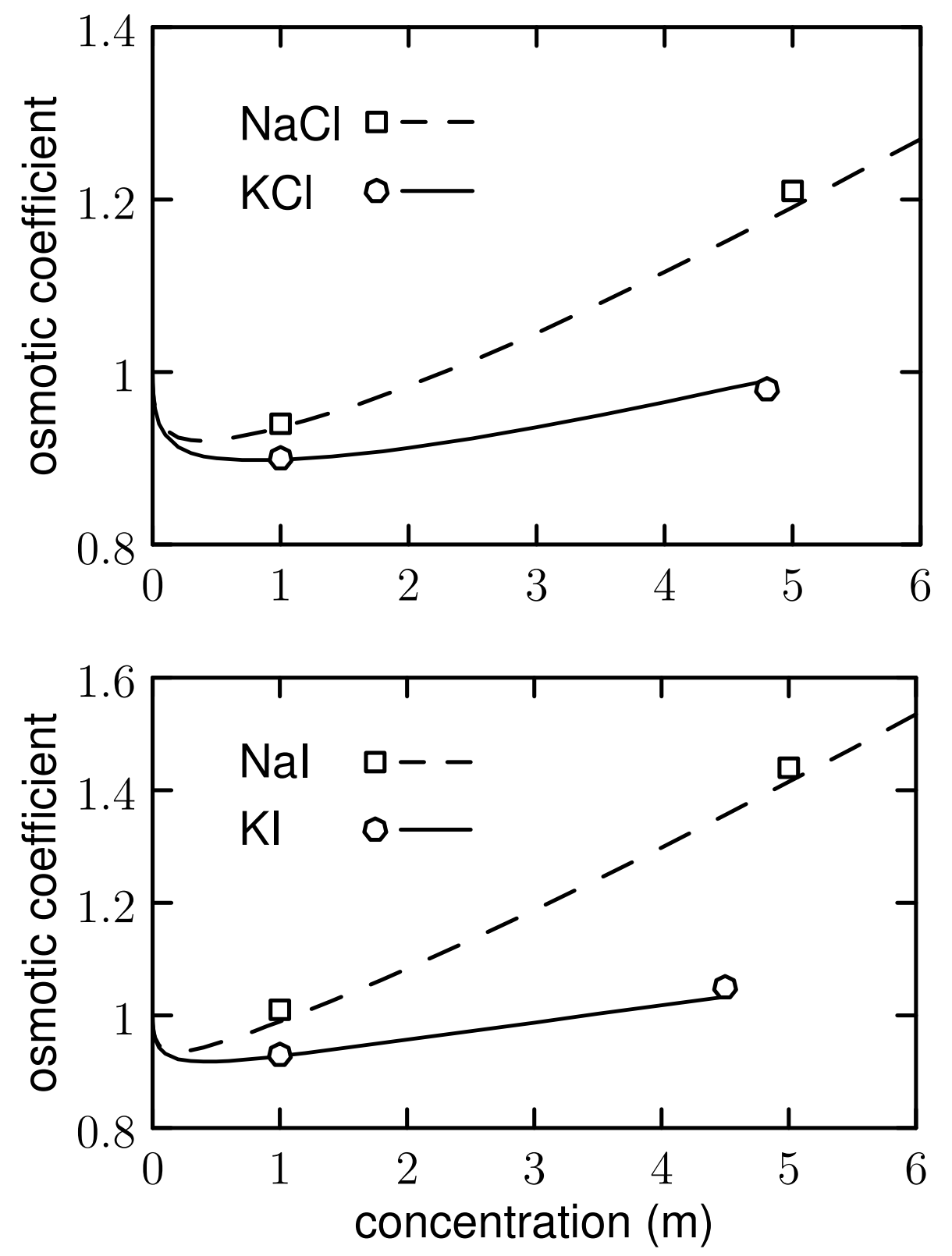

Figure 2 The osmotic coefficients of the model (symbols) and experiment[39] (lines) as a function of concentration. 

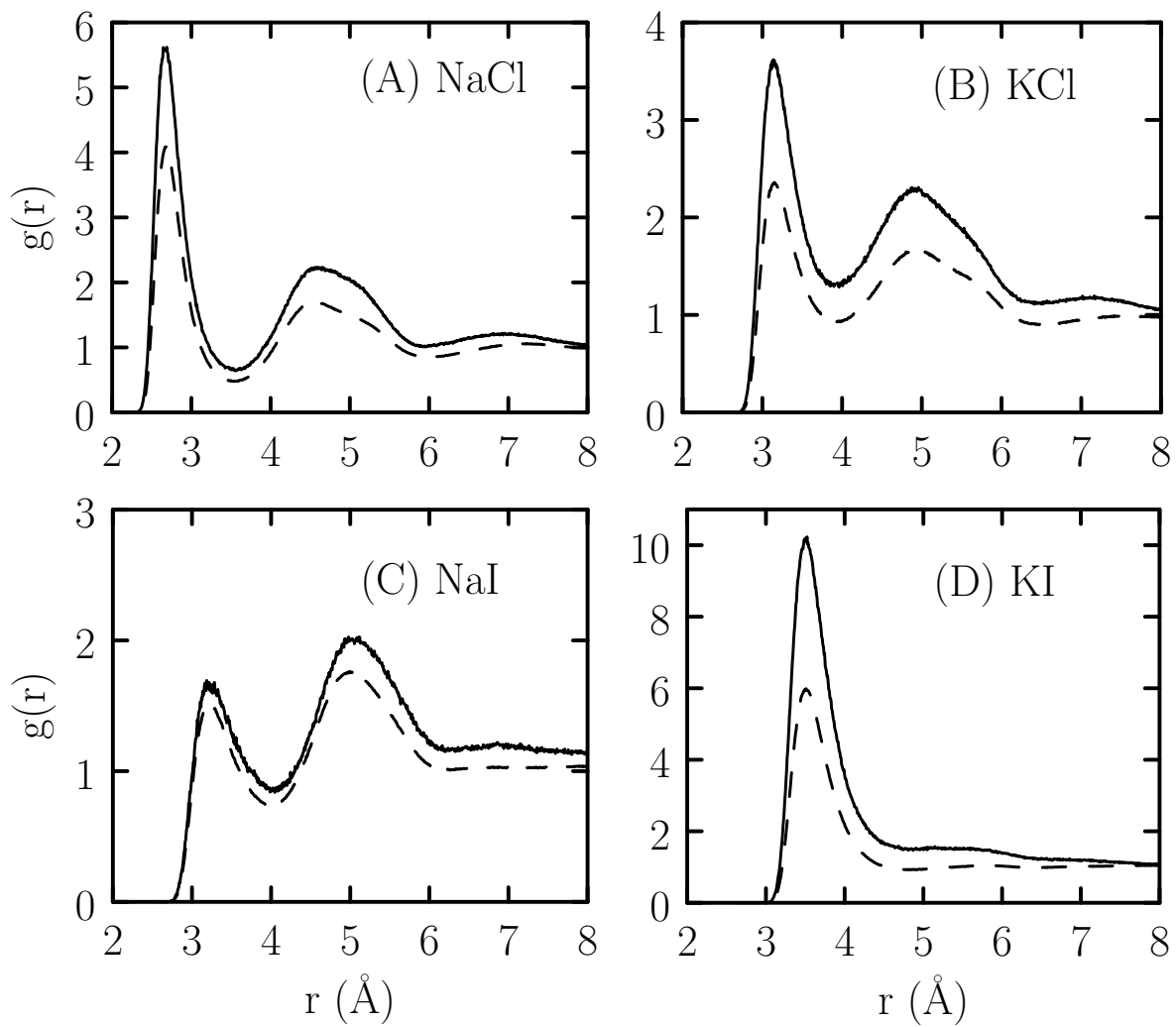

Figure 3 Pair correlation functions for ion pairs at $1 \mathrm{~m}$ (solid line) and $4.5 \mathrm{~m}$ (dashed line). 

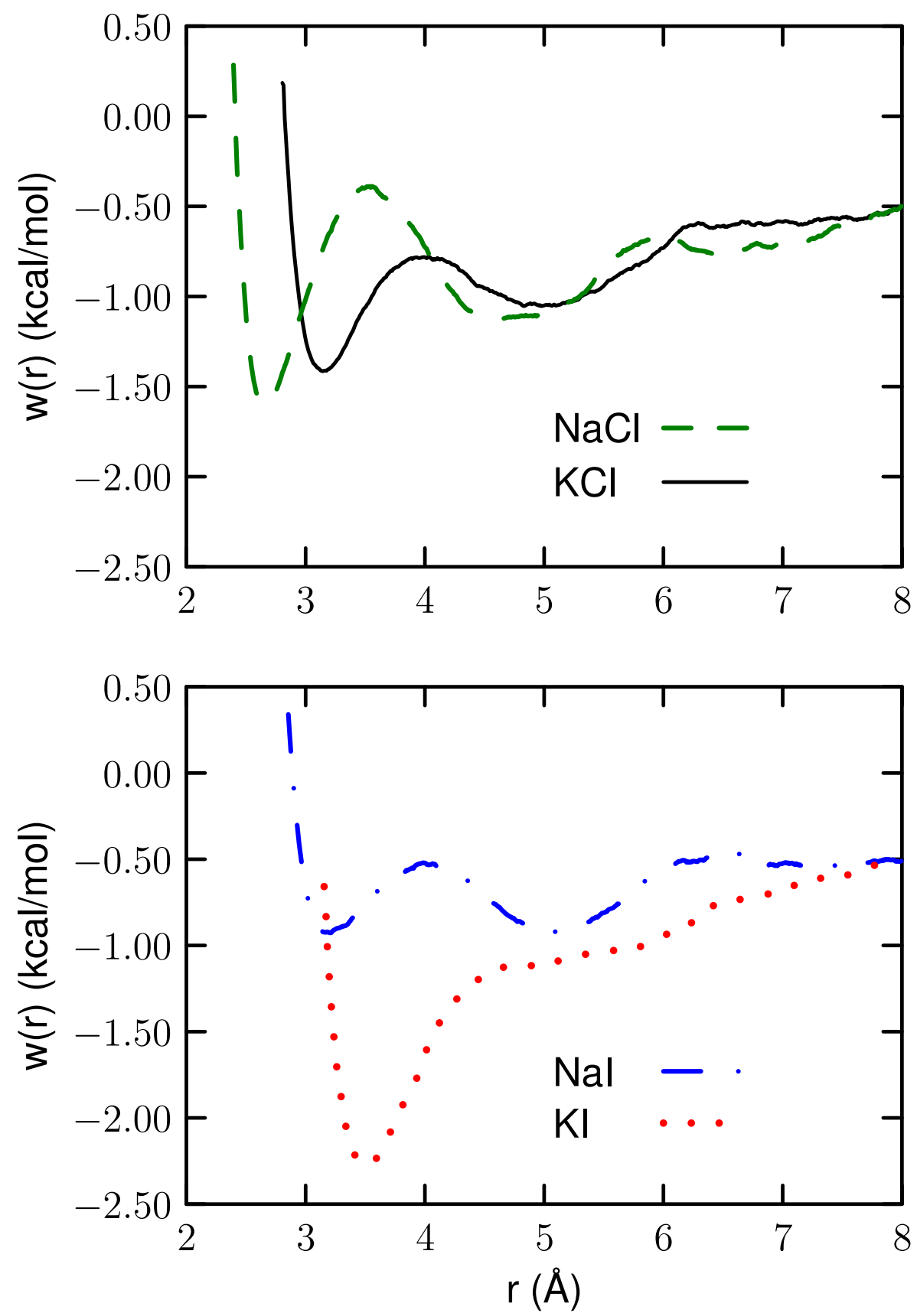

Figure 4 Potential of mean force between ion pairs for $\mathrm{NaCl}$ (green dashed line), $\mathrm{KCl}$ (black solid line), NaI (blue dot-dashed line), and KI (red dotted line). 


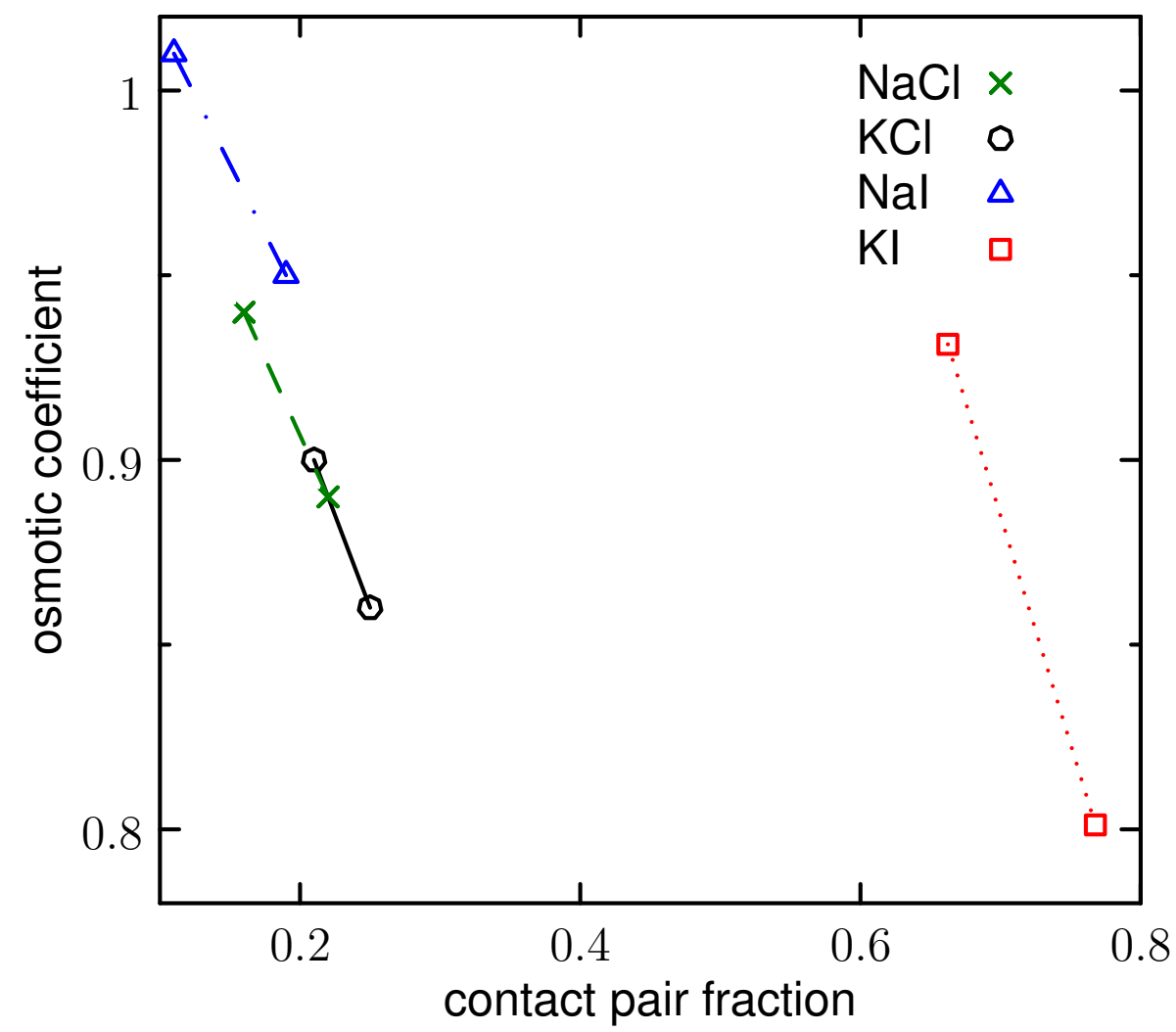

Figure 5 Osmotic coefficient as a function of contact pair fraction for models variations of the different ion pairs at $1 \mathrm{~m}$.

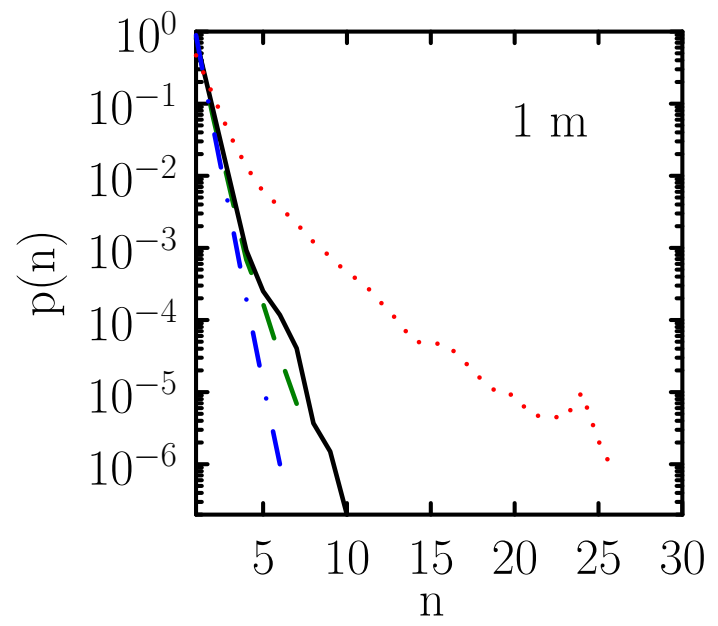

(a)

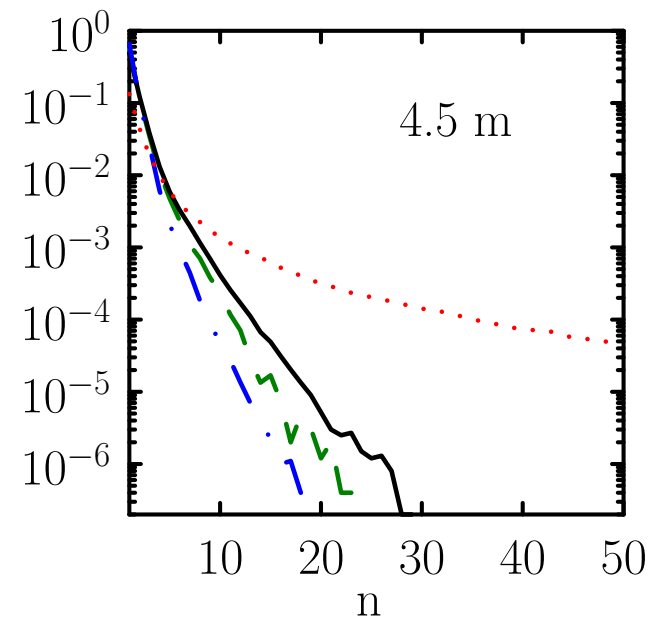

(b)

Figure 6 Ion clusters distributions for solutions of $\mathrm{NaCl}$ (green dashed line), $\mathrm{KCl}$ (solid line), NaI (blue dot-dashed line) and KI (red dotted line) at concentrations of (a) $1 \mathrm{~m}$ and (b) $4.5 \mathrm{~m}$. 


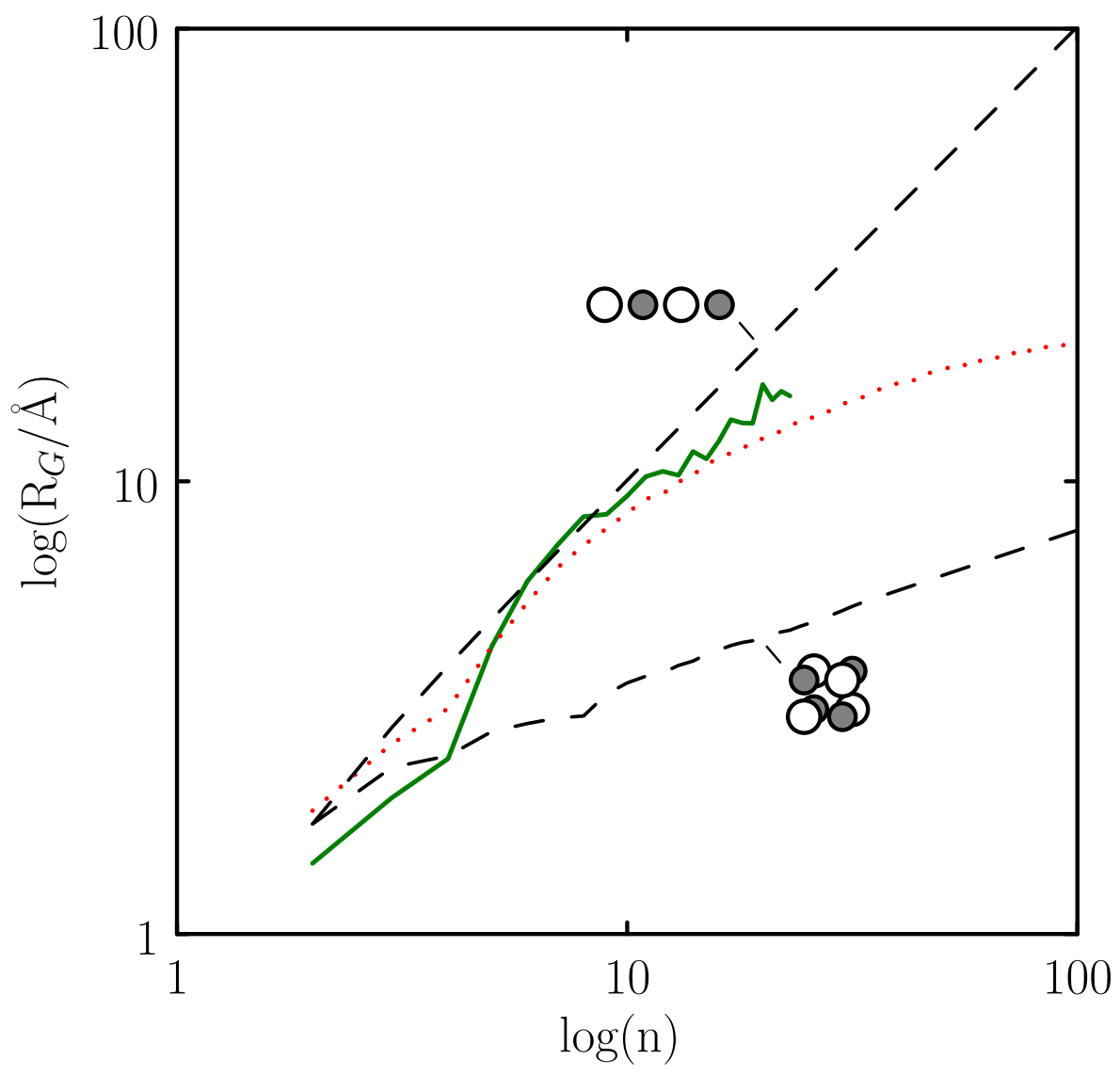

Figure 7 Radius of gyration for ion clusters of size $\mathrm{n}$ in $4.5 \mathrm{~m} \mathrm{NaCl}$ (green solid line) and KI (red dotted line) on a log-log scale. Also shown is the radius of gyration for ideal linear (upper dotted line) and ideal crystal (lower dotted line) clusters. 


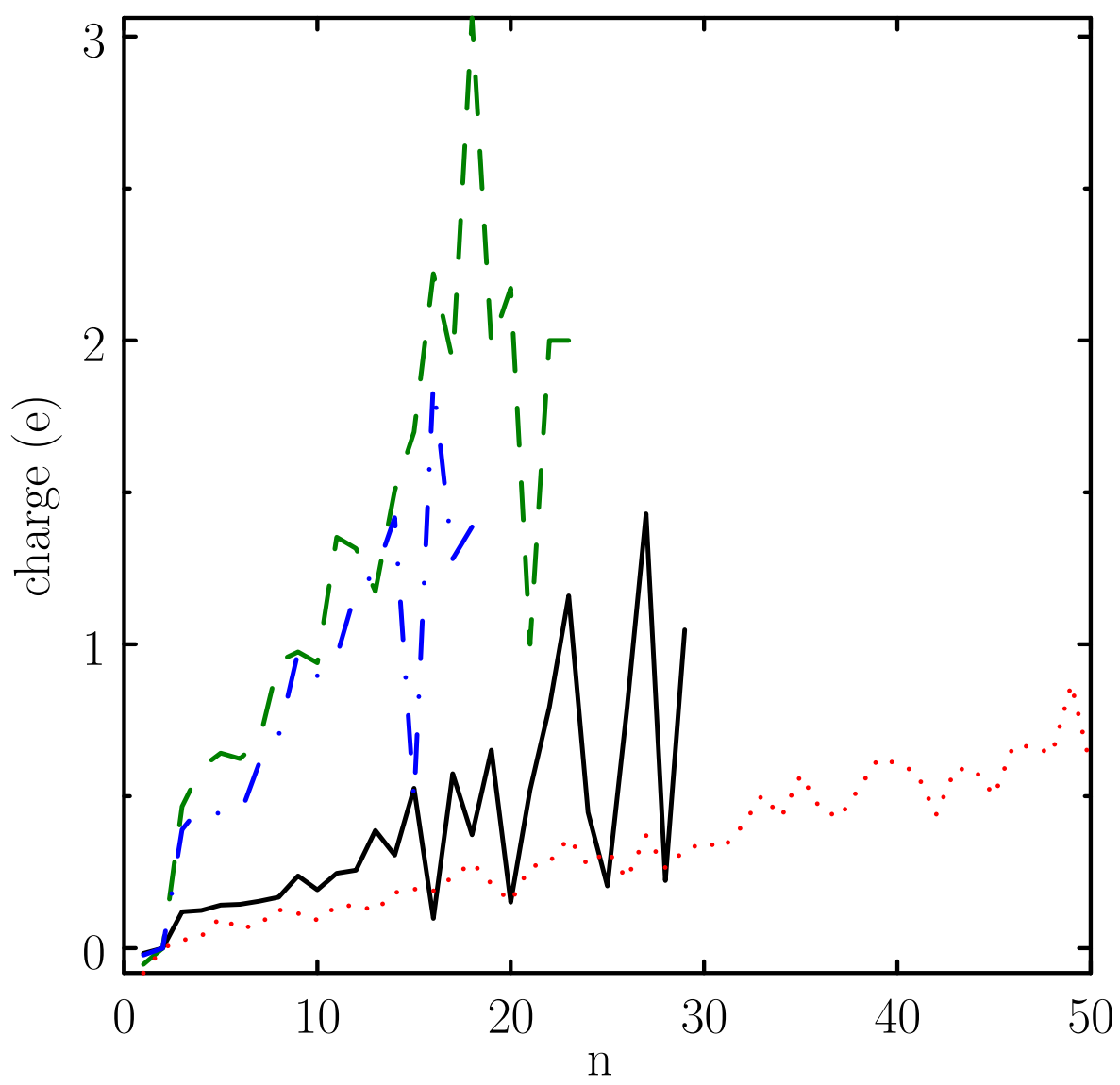

Figure 8 Formal charge of the ion clusters of size $\mathrm{n}$ in $4.5 \mathrm{~m} \mathrm{NaCl}$ (dashed green), $\mathrm{KCl}$ (solid), NaI (dot-dashed blue). and KI (dotted red line). 

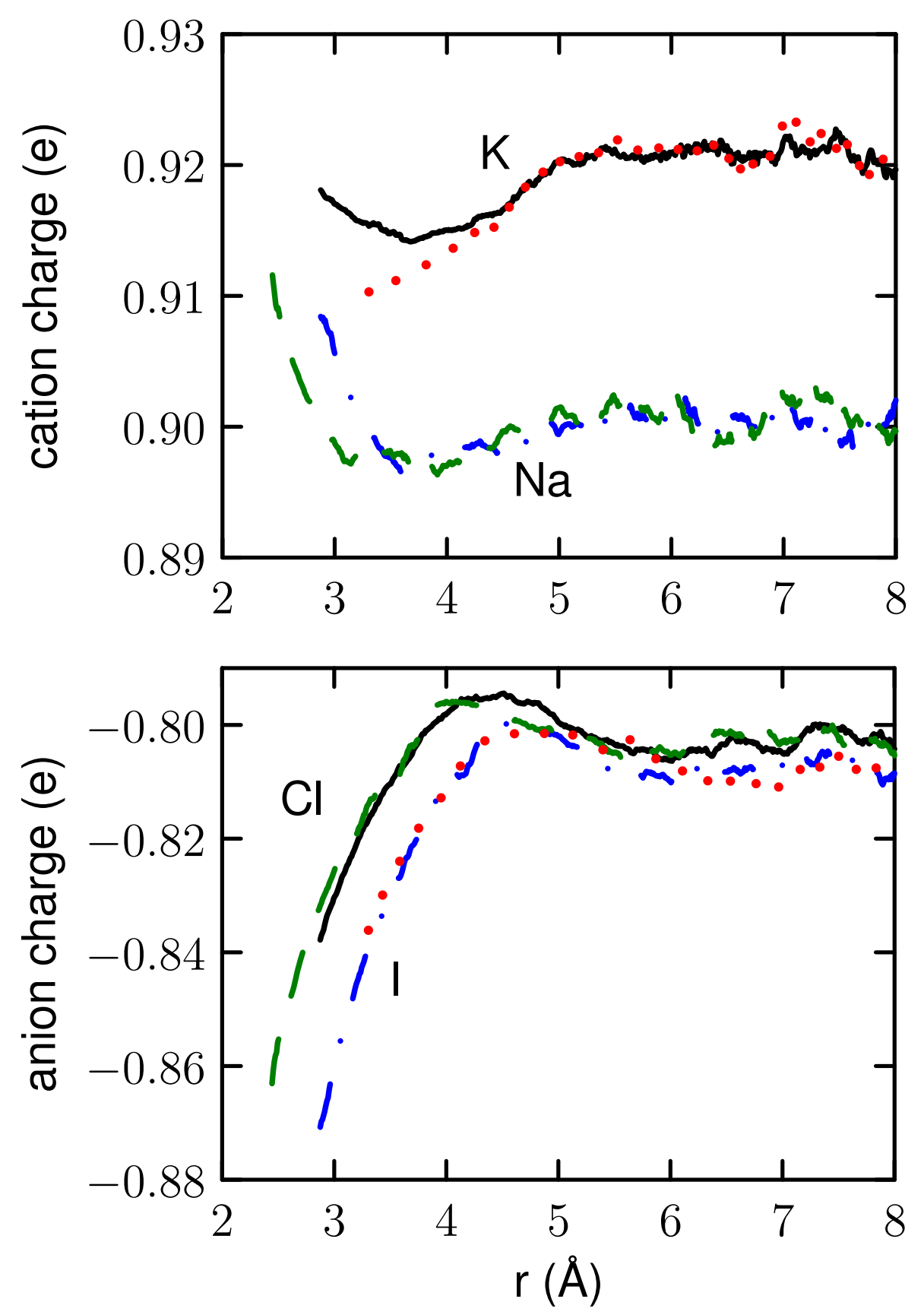

Figure 9 The cation (top) and anion (bottom) charge as a function of ion pair separation for $\mathrm{NaCl}$ (green dashed line), $\mathrm{KCl}$ (black solid line), $\mathrm{NaI}$ (blue dot-dashed line) and $\mathrm{KI}$ (red dotted line). 


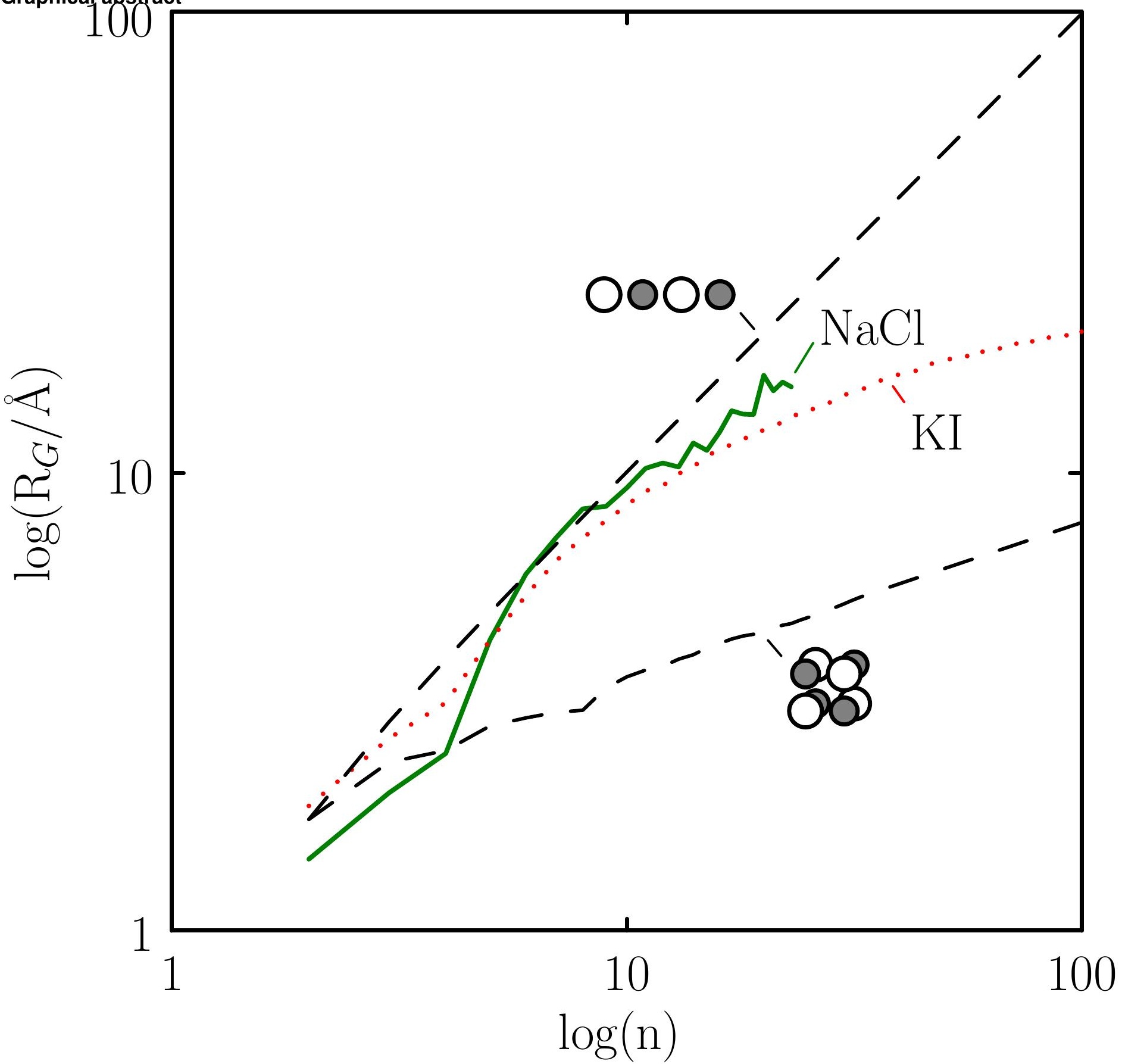

